\title{
Predictors of Hospitalization for Manic Episode in Alzheimer's Dementia: Inputs From an Inpatient Case-Control Study
}

Varsha Nandwana ${ }^{1}$, Jaskaranpreet Kaur ${ }^{2}$, Ripudaman Singh ${ }^{3}$, Sanobar Jaka ${ }^{4}$, Gagan Kaur ${ }^{5}$, Era Rawal ${ }^{6}$, Keerthika Mathialagan ${ }^{7}$, Ozge C. Amuk Williams ${ }^{8}$

1. Medicine, Lady Hardinge Medical College, Delhi, IND 2. Medicine, Dayanand Medical College \& Hospital, Ludhiana, IND 3. Internal Medicine, Jawaharlal Nehru Medical College, Wardha, IND 4. School of Global Public Health, New York University, New York, USA 5. Medicine and Surgery, Sri Guru Ram Das Institute of Medical Sciences \& Research, Amritsar, IND 6. Medicine/Cardiology, Norvic International Hospital, Kathmandu, NPL 7. Psychiatry, Sree Balaji Medical College and Hospital, Chennai, IND 8. Psychiatry, Griffin Memorial Hospital, Norman, USA

Corresponding author: Ozge C. Amuk Williams, drozgeceren@gmail.com

\section{Abstract}

\section{Objectives}

The correlates of manic episodes in dementia have not been systematically studied. The primary goal of our study is to compare the sociodemographic characteristics and psychiatric comorbidities in Alzheimer's dementia ( $\mathrm{AD}$ ) inpatients with manic episodes versus without manic episodes, and to evaluate the demographic predictors and risk factors for manic episodes in $\mathrm{AD}$ inpatients.

\section{Methods}

We conducted a case-control study using the Nationwide Inpatient Sample of 34,285 AD patients (age $\geqslant 60$ years). Subsequently, the cases i.e., $\mathrm{AD}$ inpatients with a manic episode $(\mathrm{N}=1,035)$ and the controls (without a manic episode, $\mathrm{N}=1,035$ ), were extracted using propensity-score matching based on age. The cases did not have a past psychiatric history of bipolar disorders. We used the logistic regression model to evaluate the odds ratio (OR) of association between pre-existing psychiatric comorbidities and manic episodes and evaluate the demographic predictors of manic episodes in $\mathrm{AD}$ inpatients.

\section{Results}

A higher proportion of $\mathrm{AD}$ inpatients with manic episodes were females (63.8\%), whites (85.2\%), and from low-income families below the $50^{\text {th }}$ percentile (63\%). Females were more likely to be hospitalized for manic episodes (OR 1.33; 95\% CI 1.09-1.64) than males. AD inpatients with manic episodes had a higher risk of presenting with suicidal behaviors (OR 1.88; 95\% CI 1.23-2.86). A significantly higher proportion of AD inpatients with manic episodes had comorbid tobacco use (5.3\% vs. $3.4 \%)$ and cannabis use (1.4\% vs. $0 \%$ ) compared to those without manic episodes.

Review began 07/29/2021 Review ended 08/12/2021 Published 08/20/2021

\section{() Copyright 2021}

Nandwana et al. This is an open access article distributed under the terms of the Creative Commons Attribution License CC-BY 4.0., which permits unrestricted use, distribution, and reproduction in any medium, provided the original author and source are credited.

\section{Conclusion}

Females with $\mathrm{AD}$ had a greater risk of being hospitalized for manic episodes. These patients have an $88 \%$ higher risk of suicidal behaviors during the manic presentation and have comorbid tobacco and cannabis use. Early diagnosis and management of manic episodes in at-risk $\mathrm{AD}$ patients are important to improve the quality of life (QoL) and outcomes.

Categories: Neurology, Psychiatry

Keywords: alzheimer's dementia, progressive dementia, manic episodes, hospitalization outcomes, demographic characteristics, risk factors

\section{Introduction}

Alzheimer's dementia $(\mathrm{AD})$ is a progressive neurodegenerative disease characterized by memory loss, cognitive deficits, and behavioral changes. Up until 2020, more than 5.5 million Americans, including an estimated 5.3 million people aged 65 years and older, had AD dementia, and two-thirds of them were women. $\mathrm{AD}$ is the fifth leading cause of death in the United States (US) for women and the eighth leading cause of death among men [1]. By 2021, of the 6.2 million people aged 65 and older with AD in the US, 3.8 million were women, and 2.4 million were men, and the incidence of $\mathrm{AD}$ and other dementias is projected to double by 2050 [2]. Older black and Hispanics are disproportionately more likely to have AD or other dementias than older white Americans. Data from the Chicago Health and Aging Project (CHAP) populationbased study of chronic health conditions indicate that $18.6 \%$ of blacks and $14 \%$ of Hispanics age 65 and older have $\mathrm{AD}$ compared with $10 \%$ of White older adults [3]. 
According to past studies, adults with bipolar disorder suffer from cognitive impairment with difficulties in attentional processing and executive function [4]. Several neurobiological and pathophysiological pathways are similar between $\mathrm{AD}$ and bipolar disorder, including hyper inflammation, involvement of the kynurenine pathway, increased oxidative stress, and reduced neurotrophic support [5,6]. Some studies have shown that patients with a history of bipolar disorder had an increased risk of developing dementia later in life. The prevalence of mania is notably higher in patients with dementia, $1 \%$ in the general population compared to $3.8 \%$ in dementia patients [7]. This risk is compounded by pre-existing risk factors, including genetic susceptibility, substance abuse, poor lifestyle, and medical comorbidities [5].

A history of psychiatric illness is one of the most significant factors associated with an increased risk of dementia, and depressive episodes being the most common psychiatric diagnosis [8]. A case-control study done on the Danish population found out that patients with dementia were more likely to be admitted for mania. However, this study did not establish a temporal relationship between the two illnesses [9].

It is crucial to explore the association of manic episodes in $\mathrm{AD}$ patients; since the management of behavioral and mood symptoms is one of the most challenging aspects of $\mathrm{AD}$. On average, the rate of dementia increases by $6 \%$ with every episode leading to admission for patients with bipolar disorder [10]. It is clinically important to identify the risk factors for manic episodes in patients with $\mathrm{AD}$. Early detection can improve the quality of life (QoL) among $\mathrm{AD}$ patients and also help in reducing the cost burden on the healthcare system.

While past research has established the association between dementia and major depressive disorder, limited studies have explored the relationship between dementia and bipolar disorder and/ manic episode. The goals of our study are to discern the differences in sociodemographic characteristics, psychiatric comorbidities, and substance use disorders (SUD) in AD patients hospitalized for manic episodes versus those with manic episodes. Next, we will measure the demographic predictors and risk factors for manic episodes in inpatients with $\mathrm{AD}$ and no previous history of bipolar disorder.

\section{Materials And Methods \\ Study sample}

We conducted a cross-sectional study using the Nationwide Inpatient Sample (NIS) from 2012 to 2014. The NIS is the largest inpatient database collected from 4,411 non-federal hospitals across 44 states in the US [11].

Our initial sample included 34,285 patients (age $\geqslant 60$ years) with Alzheimer's dementia (AD) and was grouped by a primary discharge diagnosis of a manic episode, unspecified. The diagnosis of a manic episode, unspecified, is given to patients who do not completely meet the diagnostic criteria for bipolar disorders and/ have no past psychiatric history of bipolar disorders. The prevalence of manic episodes in AD inpatients was $3 \%$. We used propensity score matching based on age at admission to extract the cases i.e., $\mathrm{AD}$ inpatients with manic episodes $(\mathrm{N}=1,035)$ and the controls i.e. $\mathrm{AD}$ inpatients without a manic episode $(\mathrm{N}=$ 1,035).

\section{Variables}

The demographic variables included in this study were age at admission, sex, race, and median household income. Psychiatric comorbidities were defined by various co-diagnoses in the patient's records. We included suicidal behaviors, anxiety disorders, post-traumatic stress disorders (PTSD) and psychotic disorders, and alcohol use disorders and substance use disorders (tobacco, cannabis, and stimulants) as the variables of interest [12].

\section{Statistical analysis}

We compared the distributions of demographic characteristics and psychiatric comorbidities in AD inpatients without versus with manic episodes by performing descriptive statistics and Pearson's chi-square test. Next, we used the logistic regression model to evaluate the odds ratio (OR) of association between psychiatric comorbidities and manic episodes and evaluate the demographic predictors of manic episodes in $\mathrm{AD}$ inpatients. In this regression model, we only included the variables which were statistically significant in the Pearson's chi-square test. All analyses were conducted using the Statistical Package for the Social Sciences (SPSS) version 26.0 (IBM Corp., Armonk, NY) and statistical significance was set at a two-sided P-value $<0.05$.

\section{Ethical approval}

The NIS is publicly available de-identified data with the protection of patients, physicians, and hospitalrelated information; henceforward, we were not required to take institutional review board permission for our study [11].

\section{Results}




\section{Cureus}

Our study population included 34,285 patients with AD. Among the inpatients admitted for a manic episode, the majority were females (63.8\%) and whites (85.2\%). The prevalence rate of manic episodes among inpatients also differed by socioeconomic status. Inpatients from low-income families below the $50^{\text {th }}$ percentile for median income had a higher prevalence of manic episodes (63\%)

Anxiety disorder was the most prevalent comorbidity in the study sample and there existed a nonsignificant difference in the prevalence between the groups. AD inpatients with manic episodes had a significantly higher prevalence of suicidal behaviors (7.7\% vs. $4.8 \%$ ) compared to those without manic episodes. A significantly higher proportion of $\mathrm{AD}$ inpatients with manic episodes had comorbid tobacco use (5.3\% vs. $3.4 \%)$ and cannabis use disorder (1.4\% vs. $0 \%$ ) as shown in Table 1.

\begin{tabular}{|c|c|c|c|}
\hline \multirow{2}{*}{ Variable } & \multicolumn{2}{|c|}{ Manic episode } & \multirow{2}{*}{ P-value } \\
\hline & No & Yes & \\
\hline Mean age (SD), in years & $75.6(7.7)$ & $75.6(7.7)$ & 1.000 \\
\hline \multicolumn{4}{|l|}{ Sex, in \% } \\
\hline Male & 43.5 & 36.2 & \multirow{3}{*}{0.001} \\
\hline Female & 56.5 & 63.8 & \\
\hline \multicolumn{3}{|l|}{ Race, in \% } & \\
\hline White & 89.2 & 85.2 & \multirow{4}{*}{$<0.001$} \\
\hline Black & 3.9 & 6.1 & \\
\hline Hispanic & 3.9 & 7.1 & \\
\hline Others & 2.9 & 1.5 & \\
\hline \multicolumn{4}{|l|}{ Median household income, in \% } \\
\hline Below $50^{\text {th }}$ percentile & 29.9 & 63.0 & \multirow{2}{*}{$<0.001$} \\
\hline Above $50^{\text {th }}$ percentile & 70.1 & 37.0 & \\
\hline \multicolumn{4}{|l|}{ Psychiatric comorbidities, in \% } \\
\hline Suicidal behaviors & 4.8 & 7.7 & 0.007 \\
\hline Anxiety disorders & 23.2 & 20.3 & 0.110 \\
\hline Post-traumatic stress disorder & 3.4 & 1.4 & 0.004 \\
\hline Psychotic disorders & 18.8 & 0.5 & $<0.001$ \\
\hline \multicolumn{4}{|l|}{ Substance use disorders, in \% } \\
\hline Alcohol & 4.8 & 5.3 & 0.616 \\
\hline Tobacco & 3.4 & 5.3 & 0.031 \\
\hline Cannabis & 0 & 1.4 & $<0.001$ \\
\hline Stimulants & - & - & - \\
\hline
\end{tabular}

TABLE 1: Distribution of demographics and comorbidities in Alzheimer's dementia inpatients by manic episodes

SD: standard deviation.

Females with $\mathrm{AD}$ are more likely (OR: 1.33; 95\% CI 1.09-1.64) to be hospitalized for manic episodes than males. When compared to whites, blacks had an increased odds for manic episodes, but the results were not statistically significant (OR: 1.34; 95\% CI 0.85-2.09). Other races had a non-significant negative association with manic episodes. Among socio-economic classes, the higher-income percentile was at a lower risk for manic episodes (OR: 0.51; 95\% CI 0.46-0.57). 
$\mathrm{AD}$ inpatients with manic episodes were at an increased risk of presenting with suicidal behaviors (OR: 1.88; 95\% CI 1.23-2.86) compared to those without manic episodes. Also, patients with comorbid tobacco use disorders are at higher odds for manic episodes (OR: 1.86; 95\% CI 1.03-3.38) as shown in Table 2.

\begin{tabular}{|c|c|c|c|}
\hline \multirow{2}{*}{ Variable } & \multicolumn{2}{|c|}{ Logistic regression model } & \multirow{2}{*}{ P-value } \\
\hline & Odds ratio & $95 \%$ confidence interval & \\
\hline \multicolumn{4}{|l|}{ Sex } \\
\hline Male & \multicolumn{2}{|l|}{ Reference } & \\
\hline Female & 1.33 & $1.09-1.64$ & 0.001 \\
\hline \multicolumn{4}{|l|}{ Race, in \% } \\
\hline White & \multicolumn{2}{|l|}{ Reference } & \\
\hline Black & 1.34 & $0.85-2.09$ & 0.212 \\
\hline Hispanic & 0.94 & $0.59-1.49$ & 0.780 \\
\hline Others & 0.52 & $0.24-1.13$ & 0.096 \\
\hline \multicolumn{4}{|l|}{ Median household income, in \% } \\
\hline Below $50^{\text {th }}$ percentile & \multicolumn{2}{|l|}{ Reference } & \\
\hline Above $50^{\text {th }}$ percentile & 0.51 & $0.46-0.57$ & $<0.001$ \\
\hline \multicolumn{4}{|l|}{ Psychiatric comorbidities, in \% } \\
\hline Suicidal behaviors & 1.88 & $1.23-2.86$ & 0.003 \\
\hline Post-traumatic stress disorder & 0.38 & $0.19-0.77$ & 0.007 \\
\hline Psychotic disorders & 0.03 & $0.01-0.06$ & $<0.001$ \\
\hline \multicolumn{4}{|l|}{ Substance use disorders, in \% } \\
\hline Tobacco & 1.86 & $1.03-3.38$ & 0.041 \\
\hline Cannabis & - & - & 0.999 \\
\hline
\end{tabular}

\section{TABLE 2: Predictors of a manic episode in Alzheimer's dementia inpatients}

\section{Discussion}

In this cross-sectional inpatient study of 34,285 patients with $\mathrm{AD}$ and no past diagnosis bipolar disorders, $3 \%$ had manic episodes, which are comparable to the $6 \%$ overall prevalence of late-life mania [13]. There is very limited research on mania in the elderly population since its symptoms overlap with many other organic and chronic diseases and are often erroneously interpreted as delirium [14]. It is imperative to understand the association of manic episodes in $\mathrm{AD}$ patients to draw meaningful epidemiologic and prognostic factors for acute and chronic care management of the elderly.

The patient with $\mathrm{AD}$ in our study had a mean age of 75.6 and constituted majorly by females (60.2\%) and whites (87.2\%), which is consistent with the demographic distribution from the US Census 2016 report [15]. Females are more likely to suffer from $\mathrm{AD}$ and are also more likely to experience manic episodes following $\mathrm{AD}$ possibly due to an overall higher probability of diagnosing psychiatric illness among females $[2,16]$. High-income percentile AD patients were at lower odds of getting hospitalized due to manic episodes. This finding could potentially reflect the inverse relationship between socioeconomic status and dementia diagnosis [17]. The population with higher education and higher income class is hypothesized to have built "cognitive reserve" and could be a protective factor for $\mathrm{AD}[18]$.

Race was not a statistically significant predictor of mania in $\mathrm{AD}$, although significantly limited by the health disparities among minority communities and access to quality primary and geriatric care. Prior psychotic episodes, tobacco use, and a low household income were strongly associated with manic episodes in AD. Bipolar disorder has the highest prevalence of SUD than any other psychiatric illness [19]. Past literature exploring the association between cannabis use and exacerbation of manic episodes revealed a three-fold 
increased risk of manic episodes [20]. In our study, we found a significantly higher prevalence of comorbid tobacco use and cannabis use among AD patients presenting with manic episodes. Additionally, we found a statistically significant association between $\mathrm{AD}$ inpatients presenting with suicidal behaviors than nonmanic episodes. $\mathrm{AD}$ is a known risk factor for suicidal behavior in elderly patients even years after the diagnosis [21].

It is important to further study the prevalence and the underlying risk factors for late-onset bipolar illness as it further potentiates the risk of developing dementia. Azorin et al. mentioned the term bipolar type VI i.e. the bipolarity in the context of dementia-like processes, which may help the physicians to orientate both diagnosis and treatment decisions [22].

Our study results should be considered with some limitations. Potential diagnosis of a manic episode may be masked with delirium in patients with dementia due to the overlapping nature of the presentation. As a limitation of a retrospective, observational study, we could not establish a causal relationship between AD and manic episodes. These findings from the dataset may underreport other comorbidities which are preexisting conditions/diagnoses in the patient records. The diagnoses for comorbid substance use in remission were excluded from our study but we cannot confirm the continuous/episodic use of the substance and its relationship with manic episodes. The strength of our study is that we used the NIS, which provides a population-based dataset with national representation. The results are sufficiently generalizable to the inpatient population, and there is a low risk of reporting bias since the data codes are independently reported by an individual practitioner.

\section{Conclusions}

Manic episodes in $\mathrm{AD}$, albeit relatively rare occurrence, is a significant deterrent of QoL among elderly patients. Females with $\mathrm{AD}$ were at higher risk of hospitalizing for manic episodes. Meanwhile, a higher socioeconomic status was a relatively protective factor against developing mania among AD inpatients. These at-risk $\mathrm{AD}$ patients with manic episodes have an $88 \%$ higher risk of suicidal behaviors compared to patients without manic episode presentation. Also, comorbid tobacco use and cannabis use increases the likelihood of manic episode-related hospitalization. Early diagnosis and management of manic episodes in at-risk $\mathrm{AD}$ patients are important to improve the QoL and outcomes.

\section{Additional Information}

\section{Disclosures}

Human subjects: Consent was obtained or waived by all participants in this study. Animal subjects: All authors have confirmed that this study did not involve animal subjects or tissue. Conflicts of interest: In compliance with the ICMJE uniform disclosure form, all authors declare the following: Payment/services info: All authors have declared that no financial support was received from any organization for the submitted work. Financial relationships: All authors have declared that they have no financial relationships at present or within the previous three years with any organizations that might have an interest in the submitted work. Other relationships: All authors have declared that there are no other relationships or activities that could appear to have influenced the submitted work.

\section{References}

1. Nebel RA, Aggarwal NT, Barnes LL, et al.: Understanding the impact of sex and gender in Alzheimer's disease: a call to action. Alzheimers Dement. 2018, 14:1171-83. 10.1016/j.jalz.2018.04.008

2. Alzheimer's Association: 2020 Alzheimer's disease facts and figures . Alzheimers Dement. 2020, 16:391-460. 10.1002/alz.12068

3. Alzheimer's Association: 2021 Alzheimer's disease facts and figures . Alzheimers Dement. 2021, 17:327-406. 10.1002/alz.12328

4. Robinson LJ, Ferrier IN: Evolution of cognitive impairment in bipolar disorder: a systematic review of crosssectional evidence. Bipolar Disord. 2006, 8:103-16. 10.1111/j.1399-5618.2006.00277.x

5. Diniz BS, Teixeira AL, Cao F, Gildengers A, Soares JC, Butters MA, Reynolds CF 3rd: History of bipolar disorder and the risk of dementia: a systematic review and meta-analysis. Am J Geriatr Psychiatry. 2017, 25:357-62. 10.1016/j.jagp.2016.11.014

6. Drange OK, Smeland OB, Shadrin AA, et al.: Genetic overlap between Alzheimer's disease and bipolar disorder implicates the MARK2 and VAC14 genes. Front Neurosci. 2019, 13:220. 10.3389/fnins.2019.00220

7. Lyketsos CG, Corazzini K, Steele C: Mania in Alzheimer's disease. J Neuropsychiatry Clin Neurosci. 1995, 7:350-2. 10.1176/jnp.7.3.350

8. Wetherell JL, Gatz M, Johansson B, Pedersen NL: History of depression and other psychiatric illness as risk factors for Alzheimer disease in a twin sample. Alzheimer Dis Assoc Disord. 1999, 13:47-52. 10.1097/00002093-199903000-00007

9. Nilsson FM, Kessing LV, Sørensen TM, Andersen PK, Bolwig TG: Enduring increased risk of developing depression and mania in patients with dementia. J Neurol Neurosurg Psychiatry. 2002, 73:40-4. 10.1136/jnnp.73.1.40

10. Kessing LV, Andersen PK: Does the risk of developing dementia increase with the number of episodes in patients with depressive disorder and in patients with bipolar disorder?. J Neurol Neurosurg Psychiatry. 2004, 75:1662-6. 10.1136/jnnp.2003.031773 


\section{Cureus}

11. Overview of the National (Nationwide) Inpatient Sample . (2021). Accessed: June 18, 2021: https://www.hcup-us.ahrq.gov/nisoverview.jsp.

12. NIS description of data elements . (2020). Accessed: June 18, 2021: https://www.hcupus.ahrq.gov/db/nation/nis/nisdde.jsp.

13. Dols A, Kupka RW, van Lammeren A, Beekman AT, Sajatovic M, Stek ML: The prevalence of late-life mania: a review. Bipolar Disord. 2014, 16:113-8. 10.1111/bdi.12104

14. McDonald WM: Epidemiology, etiology, and treatment of geriatric mania. J Clin Psychiatry. 2000, 61:3-11.

15. The population 65 years and older in the United States: American community survey reports . (2016). Accessed: June 28, 2021: https://www.census.gov/content/dam/Census/library/publications/2018/acs/ACS38.pdf.

16. Mental health disparities: diverse populations. (2017). Accessed: June 15, 2021: https://www.psychiatry.org/psychiatrists/cultural-competency/education/mental-health-facts.

17. Petersen JD, Wehberg S, Packness A, et al.: Association of socioeconomic status with dementia diagnosis among older adults in Denmark. JAMA Netw Open. 2021, 4:e2110432. 10.1001/jamanetworkopen.2021.10432

18. Stern Y: What is cognitive reserve? Theory and research application of the reserve concept . J Int Neuropsychol Soc. 2002, 8:448-60. 10.1017/S1355617702813248

19. Swann AC: The strong relationship between bipolar and substance-use disorder. Ann N Y Acad Sci. 2010, 1187:276-93. 10.1111/j.1749-6632.2009.05146.x

20. Gibbs M, Winsper C, Marwaha S, Gilbert E, Broome M, Singh SP: Cannabis use and mania symptoms: a systematic review and meta-analysis. J Affect Disord. 2015, 171:39-47. 10.1016/j.jad.2014.09.016

21. Serafini G, Calcagno P, Lester D, Girardi P, Amore M, Pompili M: Suicide risk in Alzheimer's disease: a systematic review. Curr Alzheimer Res. 2016, 13:1083-99. 10.2174/1567205013666160720112608

22. Azorin JM, Kaladjian A, Adida M, Fakra E: Late-onset bipolar illness: the geriatric bipolar type VI. CNS Neurosci Ther. 2012, 18:208-13. 10.1111/j.1755-5949.2011.00255.X 